\title{
EL USO DEL PARACAÍDAS EN LAS ACTIVIDADES LÚDICAS, COMO UN MEDIO PARA REFORZAR EL VALOR DE LA COOPERACIÓN
}

\author{
María de los Ángeles Monge Alvarado \\ Docente de la Universidad de Costa Rica. \\ Maureen Meneses Montero \\ Docente de la Universidad de Costa Rica.
}

Recibido 4-XII-2004 • Aceptado 9-XI-2004

\section{Introducción}

Resumen: Cuando los docentes inician actividades lúdicas con sus alumnos, siempre buscan ampliar el juego a una educación integral, donde los valores humanos tienen gran relevancia. El valor de la cooperación es uno de ellos, y a través de actividades con materiales como el paracaídas es fácilmente comprendido por los alumnos.

Se debe recordar que Vygotski (1979) hace referencia a la trascendencia del juego como factor central de aprendizaje y generador de la adaptación social, en el desarrollo que hace el niño en las actividades colectivas y sociales.

Los juegos cooperativos son propuestos para disminuir las manifestaciones de agresividad en los juegos promoviendo actitudes de sensibilización, cooperación, comunicación y solidaridad (Pérez, 1998).

Los niños juegan con otros y no contra los otros; juegan para superar desafíos u obstáculos y no para superar a los otros.

Se anexan actividades por realizar con paracaídas, los cuales pueden ser también implementados con materiales como sábanas o telas grandes, en caso de no tener facilidad para conseguir el material original.

Palabras clave: Actividades lúdicas, juegos en educación, fomento de valores, procesos de enseñanza aprendizaje, cooperación, paracaídas.
El juego es la vida de la infancia, es la expresión del niño, es su instrumento de conocimiento. Se vincula con la experiencia de la vida que el niño intenta repetir, dominar o negar con el fin de organizar su mundo interior en relación con el exterior (Osorio, 2000).

Esta actividad innata del ser humano es producto de una experiencia placentera, Lacayo y Coello (1992) afirman que como resultado de un compromiso en particular.

Lázaro (1995) cita a Vygotski el cual hace referencia a la trascendencia del juego como factor central de aprendizaje y generador de la adaptación social en el desarrollo que hace el niño en las actividades colectivas y sociales.

Esa actividad requiere de un tiempo, un espacio y un marco de seguridad en la escuela; sin embargo, comenta Lázaro (1995) que el niño desde la temprana infancia hace uso del juego simbólico y pone de relieve su importancia para que organice sus conocimientos sobre el mundo y sobre los otros.

El juego representa un aspecto esencial en su madurez en cuanto está ligado al desarrollo del conocimiento, la afectividad, 


\begin{abstract}
When teachers initiate playful activities with their students, they always seek to extend the game to an integral education, where human values have great relevancy. The value of cooperation is one of them and, across activities with materials like the parachute, the value is easily understood by the pupils. It is necessary to remember that Vygotski (1979) refers to the transcendence of the game as both a central factor of learning and a generator of social adjustment in the development that child undergoes in collective and social activities. Cooperative games are proposed to diminish the manifestations of aggressiveness in games that promote attitudes of sensibility, cooperation, communication and solidarity (Perez, 1998). Children play with others and not against others; they play to overcome challenges or obstacles and not to overcome others. Parachute activities are annexed which can be implemented also by materials like sheets or long fabrics, in case of difficulty getting the original material.
\end{abstract}

Key words: Playful activities, games in education, promotion of values, processes of education learning, cooperation, parachute. la motricidad y la socialización del niño. Osorio (2000) comparte su teoría acotando que al niño se le permite resolver problemas, tomar una decisión válida, sentir éxito personal e incrementar su voluntad para hacer frente a la vida.

El juego lo describe Vygotski (1979) como trascendental para el desarrollo del niño y asegura que no es un rasgo predominante en la infancia sino un factor básico en el desarrollo; donde las actividades promueven la auto confianza, la creatividad, y en la mayoría de los casos, la cooperación entre los jugadores (Orlick, 1990).

Todo lo anterior debido a que por naturaleza, a los niños les gusta investigar, manipular, transformar y crear. Esa creación se limita muchas veces a transformar una cosa en algo diferente.

Al jugar, el niño estructura y evoluciona en su personalidad. El carácter competitivo, participativo, comunicativo y agonista va adaptándose a los rasgos que rigen esta personalidad. El niño siente el deseo de ejercer un control y dominio total sobre los demás de establecer una comunicación y relación con los que lo rodean por medio de su propio cuerpo y de crear una fantasía liberadora; y encuentra la posibilidad de realizar estos deseos en la actividad lúdica, lo que la define como auténtica expresión del mundo del niño.

El niño actúa en forma positiva en el aspecto social al compartir; en el afectivo ya que se conoce más a sí mismo y a los demás y en el cognoscitivo pues desarrolla su intelecto y destrezas.

El movimiento usualmente asociado con los juegos es el objetivo, cuyo propósito es funcional, no expresivo. El objetivo puede ser definido como un movimiento para llevar a cabo una tarea de práctica (patear un balón o saltar un obstáculo). En contraste, el movimiento expresivo lleva un pensamiento o sentimiento. El cuerpo es usado para la comunicación.

Además, las situaciones de los juegos son raramente las mismas. El ambiente es siempre cambiante. Los jugadores ajustan 
constantemente los patrones de movimientos usados. El desarrollo de la versatilidad significa ser capaz de manejar eficientemente y efectivamente el cuerpo mientras se adapta constantemente a nuevas situaciones. Los movimientos de los juegos no son estereotipos.

Por lo tanto, los juegos ofrecen experiencias, las cuales deben ser incorporadas dentro del programa total de la educación física para dar a los estudiantes una amplia gama de oportunidades.

\section{Valores y actitudes en las actividades lúdicas}

Para el Ministerio de Educación Pública (1996) los valores son indispensables para el desarrollo integral del ser humano, ellos se manifiestan en las actitudes, comportamientos y las acciones cotidianas.

Son muchos los contenidos y significados que encierra el término valor; humanísticamente hablando se entiende por valor a lo que hace que un hombre sea tal, sin lo cual perdería su humanidad o parte de ella. El valor se refiere a una excelencia o perfección. Por ejemplo, se considera un valor decir la verdad, ser honesto, ser responsable, sincero. Al implementar los valores se desarrolla la humanidad de la persona, mientras que el contravalor la despoja de esa cualidad. (http://www.gruppaz. org/valores.htm)

La primera infancia y la niñez se caracterizan por la interacción con otras personas, las cuales proporcionan información social y de conductas que el niño y la niña aprenden y luego reproducen en situaciones diversas.

Cuando el individuo se encuentra con "él mismo y con el otro" conlleva una interacción de respeto y de entendimiento mutuo, permitiendo hacer relaciones y manifestando sentimientos propios de su cultura.

Desde el punto de vista socio-educativo, los valores son pautas que orientan el comportamiento humano hacia la transformación social y la realización de la persona. Son guías que dan determinada orientación a la conducta y a la vida del individuo y de cada grupo social. (http://www.gruppaz. org/valores.htm)

Los padres de familia y los educadores son responsables de fomentar y fortalecer estas relaciones en la búsqueda de una formación equitativa y solidaria del educando.

Cabe destacar que los ambientes a los que los niños hoy día se ven expuestos representan grandes preguntas que definen su personalidad, las cuales deben ser valoradas con respuestas positivas y negativas, pero formadoras, los ambientes hostiles, las injusticias económicas y sociales, el sexismo, los derechos a la libre expresión, el disfrute del ocio, entre otros, son parte de estas interrogantes.

Educar al hombre en los valores humanos es educarlo para que se oriente en el valor real de las cosas. La Declaración Universal sobre los Derechos Humanos de la Organización de las Naciones Unidas recoge el común sentir de los hombres que reconocen los valores que dignifican y acompañan la existencia de cualquier ser humano (http://www.ecojoven.com/uno/05/ valores2.html)

Hablar de valores humanos significa aceptar al hombre como el supremo valor entre todas las realidades humanas, y que no debe supeditarse a ningún otro valor terreno, dinero estado, ideología.

Y si el "mundo de los valores" puede servir de guía para la humanidad en sus aspiraciones de paz y fraternidad, debe servir también de guía al individuo en sus deseos de autorrealización y perfeccionamiento. (http://www.ecojoven.com/uno/05/ valores2.html)

Es mediante el juego o la actividad lúdica que se puede rescatar o reforzar los valores humanos que se han ido perdiendo a lo largo de las generaciones modernas, como por ejemplo la cooperación. 
El objetivo constante de la cooperación es el beneficio mutuo en las interrelaciones humanas; se fundamenta en el principio del respeto mutuo. El valor, la consideración, el cuidado y la participación proporcionan un fundamento a partir del cual puede desarrollarse el proceso de la cooperación. (http://www.livingvalues.net/ espanol/valores/libertad.htm)

La cooperación es posible cuando se es sincero y generoso; esto hace a la persona ser digna de recibir la cooperación de todos. Si uno tiene fe y confianza en los demás, eso, en retorno, construye la fe y confianza en ellos; lo que produce un ambiente de enriquecimiento, respeto, apoyo y solidaridad.

Cooperar es responsabilidad de todos, es necesario tener valor y fortaleza interna. Cada persona debe mantener la sinceridad y una actitud positiva y el grupo reconocerá el rol que tiene en él. Los pensamientos positivos crean sentimientos de cooperación en la mente de los demás. En la cooperación viene un cúmulo de buenos deseos hacia los integrantes y la tarea, así el ambiente se vuelve positivo y sutil. (http://www.livingvalues. net/espanol/valores/libertad.htm)

La cooperación genera paciencia al paso del tiempo y del acontecer de los hechos. El tiempo es el mejor compañero ya que brindará la oportunidad de obtener lo mejor y lo necesario en el momento adecuado.

\section{Juegos cooperativos}

Los juegos cooperativos son propuestas que buscan disminuir las manifestaciones de agresividad en los juegos promoviendo actitudes de sensibilización, cooperación, comunicación y solidaridad (Pérez, 1998).

Los niños juegan con otros y no contra los otros; juegan para superar desafíos u obstáculos y no para superar a los otros.

Los juegos siempre deben procurar buscar la participación de todos, sin que nadie quede excluido, independientemente de las características, condiciones, experiencias previas o habilidades personales; donde la propuesta y el clima placentero que genera están orientados hacia metas colectivas y no hacia metas individuales; deben centrarse en la unión y la suma de aportes individuales y no en el "unos contra otros".

Los juegos deben facilitar el proceso de crear: crear es construir y para construir, la importancia del aporte de todos es fundamental. Si las reglas son flexibles, los participantes pueden contribuir a reformularlos; los juegos se pueden adaptar al grupo, a los recursos, al espacio disponible y al objetivo de la actividad. (Pérez, 1998).

La cooperación es una alternativa que puede ayudar a solucionar problemas y conflictos; si el juego tiene presente los valores de solidaridad y cooperación, podemos experimentar el poder que tenemos cada uno de nosotros para proponer colectivamente soluciones creativas a los problemas que nos presenta la realidad en que vivimos, y mejorar la calidad de vida.

Como parte de los juegos cooperativos se introducen los paracaídas como material que permite gran flexibilidad en las actividades, y como elemento ideal para fomentar la cooperación.

El paracaídas es un material que sorprende a niños y grandes, ya que llama mucho la atención y reúne en poco tiempo a gran cantidad de gente alrededor.

Entre los objetivos que se persiguen al trabajar con este material están los siguientes:

- Aumentar las capacidades básicas de la fuerza, principalmente al nivel de brazos, hombros y piernas, así como también la flexibilidad de articulaciones como la del hombro y la cadera.

- Fortalecer valores y actitudes como la participación, cooperación, trabajo en equipo. 
- Fomentar en los niños el jugar mejor con otros que contra otros.

- Sensibilizar a los participantes que deben superar desafíos y no superar a los otros.

- Fomentar en los participantes que deben sentirse libres para crear, libres de la exclusión.

\section{Tipos de paracaídas}

Existen varios tipos de paracaídas. El tradicional se puede conseguir reciclando uno viejo, o intentar confeccionarlo con trozos de tela de seda; comprarlo en empresas comerciales de juguetes, deportivas o de psicomotricidad. El otro tipo es el rectangular que se puede confeccionar con una pieza de tela de algodón, con dimensiones de 18 a 28 pulgadas de ancho por 6 a 10 pies de largo, se le forma ruedo en las orillas. En las partes anchas se hace un canal para insertar un tubo de P.V.C. o de aluminio, para formar agarraderas firmes, estos pueden tener un diámetro de $1 / 2$ pulgada para niños menores o de $3 / 4$ de pulgada para los mayores. Los extremos se cosen para que el tubo no se salga.

\section{Instrucciones}

Se inician las actividades colocando el paracaídas en el suelo, bien extendido y los participantes se sentarán cómodamente alrededor del mismo. Cuando se concluye la actividad, el paracaídas se pondrá en la posición antes descrita.

Orlick (1986) invita a que si se hace de esta forma, todos los participantes escucharán las instrucciones claramente y podrán verse.

La llamada "posición de frutero" es la que se sugiere que se lleve a cabo entre actividades, esta consiste en tomar el paracaídas con las dos manos a la altura del pecho del participante de menor estatura y dejar que el centro del material toque el suelo, y así se forma el frutero.

Si la actividad no se ha concluido por los participantes, el juego no se debe detener.

Las actividades deben ser planeadas según su grado de intensidad, de tal modo que los participantes no se cansen. Si el paracaídas se sacude durante mucho tiempo puede presentarse la fatiga.

Otras de las indicaciones de Orlick (1986) es que cuando se practican actividades que envuelven a muchas personas pasando por encima o debajo del paracaídas, se debe empezar con grupos pequeños y en velocidades lentas para que se comprenda la idea del juego y no ocasionar algún accidente.

Para que las actividades se realicen fluidamente es necesario que los participantes entiendan el trabajo en equipo. El lenguaje por utilizar debe ser claro y sencillo. Las formas de tomar el paracaídas pueden variar: con las palmas hacia arriba, palmas hacia abajo y los dedos se encuentran sujetos al borde o una palma hacia abajo y la otra hacia arriba.

El primer paso es aprender a INFLAR EL PARACAÍDAS, esto implica: de cuclillas alrededor del paracaídas y tomándolo con ambas manos, a la señal; todos los participantes se levantan y extienden los brazos sobre sus cabezas levantando el paracaídas. Este se llenará de aire y parecerá un hongo gigante.

Expertos con este tipo de trabajo recomiendan algunas normas de seguridad para cuando se están practicando este tipo de actividades, como por ejemplo: tomar el paracaídas y darle unas cinco vueltas al borde de la tela, mantener la postura correcta en cada uno de los movimientos que se realizan, se debe evitar todo tipo de juego brusco y majar el paracaídas.

Preferiblemente se deben realizar estas actividades en terrenos no ásperos, puede ser en áreas verdes o piso de madera. 


\section{Actividades}

A continuación de detallan algunas de las actividades que recomiendan Orlick (1986) y Bolaños (2000) y que son puestas en práctica para fomentar la cooperación.

\section{Centrífuga}

Objetivo

- $\quad$ Favorecer la cooperación en grupo.

\section{Participantes}

A partir de los 7 años.

\section{Materiales}

Un paracaídas.

\section{Organización}

En círculo, sentados y tomando el paracaídas.

\section{Área por utilizar}

Espacio amplio, libre de obstáculos.

\section{Descripción}

Dar vueltas con un paracaídas. El que empieza el juego se tira hacia atrás y a la derecha sin soltar el paracaídas. Los demás le imitan siguiendo el movimiento en forma continua uno por uno, como si fuera una ola. Se consigue un movimiento circular ondulante.

\section{Pelota rodante}

\section{Objetivos}

- Desarrollar coordinación manual.

- $\quad$ Fortalecer el trabajo en equipo.

\section{Participantes}

A partir de los 8 años.

\section{Materiales}

Un paracaídas y un balón de hule o una bola de playa.

\section{Organización}

De pie tomando el paracaídas.

\section{Área por utilizar}

Espacio amplio, libre de obstáculos.

\section{Descripción}

Consiste en hacer rodar una pelota por el borde externo del paracaídas. Los participantes toman el paracaídas a la altura de la cintura con el balón sobre él, zarandean el paracaídas hasta conseguir que la onda traslade el balón con un recorrido circular, evitando que caiga.

\section{A volar con el paraguas gigante}

\section{Objetivos}

- Desarrollar la coordinación auditiva.

- Desarrollar la fuerza de los miembros superiores.

- $\quad$ Fortalecer el trabajo en equipo y la socialización.

\section{Participantes}

A partir de los 7 años.

\section{Materiales}

Un paracaídas.

\section{Organización}

De pie tomando el paracaídas.

Área por utilizar

Espacio amplio, libre de obstáculos.

\section{Descripción}

A la $1^{a}$ orden del profesor, se levanta el paracaídas hasta la cintura. A la $2^{\text {a }}$ orden se elevan con fuerza los brazos y al $3^{\circ}$ silbato, se suelta el paracaídas todos a la vez y se rota dos pasos a la derecha; la segunda vez que se realiza el lanzamiento se hace lo mismo pero el desplazamiento es hacia la izquierda.

\section{Variante}

Una variante sería que todos los participantes corrieran hacia el centro para dejarse cubrir por el paracaídas. Otra variante sería que uno de los participantes 
dé la orden de levantar y soltar y luego gritará: me cambio por... "nombre de un(a) compañero(a), y en ese instante antes de que les cubra el paracaídas o caiga al suelo, los dos participantes deberán cambiar sus posiciones. Como tercera variante sería sin soltar los extremos del paracaídas, levantar los brazos hacia arriba y elevar el paracaídas, en ese momento corren todos hacia el centro del círculo cerrando la copa del paracaídas y formando un hongo.

\section{El ratón y el gato}

Objetivos

- Fortalecer el trabajo en equipo la solidaridad y la cooperación.

\section{Participantes}

A partir de los 7 años.

\section{Materiales}

Un paracaídas.

\section{Organización}

De pie tomando al paracaídas.

\section{Área por utilizar}

Espacio amplio, libre de obstáculos.

\section{Descripción}

Los participantes toman el paracaídas. Uno de los participantes (el gato) debe ubicarse en el centro del paracaídas por encima de él (es decir pisándolo); el resto de los participantes debe realizar fuertes movimientos del paracaídas para provocar el máximo número de ondas posibles. En ese momento otra persona (el ratón) se mete por debajo del paracaídas y escapa del gato que está encima y que intenta atraparlo. Debido al número de cuerpos que provocan las ondas es bastante difícil apresarlo, sobre todo si el ratón va muy agachado.

Todo el grupo debe ayudar al ratón a huir. Se debe insistir en que el gato siempre camine, que nunca salte, porque puede golpear o pisar al ratón.

\section{Variante}

Dependiendo del tamaño del paracaídas puede haber más de un ratón o gatos.

\section{Lanzar objetos}

Objetivos

- Desarrollar coordinación manual.

- Fortalecer el trabajo en equipo.

\section{Participantes}

A partir de los 8 años.

\section{Materiales}

Un paracaídas, balones de hule, bolas de playa, bolas de tenis, estereofón, objetos livianos.

\section{Organización}

De pie tomando el paracaídas.

\section{Área por utilizar}

Espacio amplio, libre de obstáculos.

\section{Descripción}

Con el paracaídas paralelo al suelo, se introducen los diferentes objetos que se van a lanzar. Los participantes mueven el paracaídas tratando de que los diferentes objetos se eleven lo máximo para apañarlos en su regreso con el paracaídas. El juego termina cuando no queda en el paracaídas ningún objeto que lanzar.

\section{Variante}

Lanzar los objetos en un tiempo determinado con anterioridad, se anota un punto por cada objeto que aún permanezca en el paracaídas al cabo de ese tiempo.

\section{Lanzar objetos en equipo}

Objetivos

- Desarrollar coordinación manual.

- Fortalecer el trabajo en equipo.

\section{Participantes}

A partir de los 8 años. 


\section{Materiales}

Un paracaídas, balones de hule, bolas de playa, bolas de tenis, estereofón, objetos livianos.

\section{Organización}

De pie tomando el paracaídas.

\section{Área por utilizar}

Espacio amplio, libre de obstáculos.

\section{Descripción}

Los participantes se dividen en dos equipos, cada uno tiene un nombre, el elegido por sus integrantes. Los de un equipo se acomodan junto al paracaídas, los del otro equipo se colocan afuera con otros objetos que van a lanzar. Con el paracaídas paralelo al suelo, se introducen los diferentes objetos. El equipo 1 comienza a hacer movimientos con el paracaídas tratando de que los diferentes objetos se eleven lo máximo posible para recogerlos en su caída con el paracaídas. Si algún objeto se sale fuera del paracaídas, los del equipo 2 pueden devolverlo al paracaídas, lanzándolo desde donde lo cogieron. Para intercambiar los papeles de los grupos el profesor puede decir: "equipo 1, al paracaídas", en ese instante los dos grupos están lanzando objetos, tras una pausa dirá: "equipo 2, fuera", momento en el cual sus integrantes dejan de agarrar el paracaídas y se ocupan de recoger los objetos que se salen de él. El juego termina cuando no queda en el paracaídas ningún objeto por lanzar.

\section{Variante}

Lanzar los objetos en un tiempo determinado con anterioridad, se anota un punto por cada objeto que aún permanezca en el paracaídas al cabo de ese tiempo.

\section{La piedra y las pepitas de oro}

\section{Objetivo}

- $\quad$ Favorecer la cooperación en grupo.

\section{Participantes}

A partir de los 7 años.

\section{Materiales}

Un paracaídas, seis balones, cinco iguales y uno diferente.

\section{Organización}

De pie tomados del paracaídas.

\section{Área por utilizar}

Espacio amplio, libre de obstáculos.

\section{Descripción}

Se coloca el paracaídas extendido en el suelo, se sitúan en él seis balones, uno de los cuales presenta una característica especial, ya sea su tamaño, su color, entre otros, que le diferencia del resto. Ese balón es la piedra y el resto son las pepitas de oro. Los participantes inician movimientos con el paracaídas tratando de que las pepitas se introduzcan por el agujero central del paracaídas y evitando que la piedra lo haga. Por cada pepita que se introduzca por el agujero central se anota un punto. El juego termina cuando la piedra se introduce por el agujero o se sale del paracaídas.

\section{Variante}

Definir un tiempo de juego. Instalar más piedras o más pepitas. El juego termina cuando no queda ninguna piedra en el paracaídas.

\section{El velero}

\section{Objetivo}

- $\quad$ Favorecer la cooperación en grupo.

\section{Participantes}

A partir de los 7 años.

\section{Materiales}

Un paracaídas, un balón.

\section{Organización}

En círculo de pie tomando el paracaídas. 


\section{Área por utilizar}

Espacio amplio, libre de obstáculos.

\section{Descripción}

Se extiende el paracaídas en el suelo, se coloca una pelota. Los participantes inician el movimiento de olas tratando de que la pelota se encaje por el agujero y sorteando que se salga fuera del paracaídas. La intención es introducir la pelota por el orificio, evadiendo que salte del paracaídas.

\section{Variante}

Cada vez que el balón se meta en el agujero es un punto y cada vez que se salga es un punto en contra. Se juega en sets de cinco puntos. Igual al anterior pero en un lapso determinado. Se cuenta las veces que el balón se introduce por el boquete.

\section{El lobo y la liebre}

\section{Objetivo}

- $\quad$ Favorecer la cooperación en grupo.

\section{Participantes}

A partir de los 7 años.

\section{Materiales}

Un paracaídas y dos balones medianos de diferente color.

\section{Organización}

En círculo tomando al paracaídas.

\section{Área por utilizar}

Espacio amplio, libre de obstáculos.

\section{Descripción}

Se extiende el paracaídas en el suelo, se colocan las dos pelotas: el lobo y la liebre. Los participantes emprenden el movimiento de olas tratando de que la pelota que representa a la liebre se introduzca por el agujero central antes de la que representa al lobo y evitando que cualquiera de las dos se salga fuera del paracaídas. Se gana el juego si la liebre se introduce en la madriguera antes que el lobo la atrape o que se meta en su casa. Se pierde si primero se introduce el lobo y después la liebre. Se pierde si se salen del paracaídas.

\section{Variantes}

Introducir varios lobos para aumentar el grado de dificultad del juego.

\section{Que pasen los ...}

\section{Objetivo}

- $\quad$ Favorecer la cooperación en grupo.

\section{Participantes}

A partir de los 7 años.

\section{Materiales}

Un paracaídas.

\section{Organización}

En círculo tomando el paracaídas.

\section{Área por utilizar}

Espacio amplio, libre de obstáculos.

\section{Descripción}

Los participantes se colocan tomando el paracaídas. En un momento se eleva el paracaídas y el profesor dice ..."que pasen los de "X" color, equipo de fútbol, color de las diferentes prendas de vestir, entre otros, los que coinciden con el nombrado cambian de sitio por debajo del paracaídas $\mathrm{y}$ antes de que se desinfle.

\section{Variantes}

Llamar dos colores a la vez. Ambos se cruzan por debajo del paracaídas. Designar dos colores a la vez. Los del primer color se cambian de sitio por debajo del paracaídas y los del segundo deben hacerlo alrededor de él. Si el profesor dice: “Todos abajo!", el paracaídas se infla y todos los participantes se meten debajo procurando que cuando éste descienda nadie quede al descubierto. 


\section{El lago de las estatuas}

\section{Objetivo}

- $\quad$ Favorecer la cooperación en grupo.

\section{Participantes}

A partir de los 7 años.

\section{Materiales}

Un paracaídas, aros.

\section{Organización}

En círculo tomando el paracaídas.

\section{Área por utilizar}

Espacio amplio, libre de obstáculos.

\section{Descripción}

Los participantes están colocados alrededor del paracaídas. Cada uno de ellos es un color en este orden: azul, rojo, verde, amarillo. Bajo el paracaídas se colocan varios aros. Son las piedras del lago de las estatuas. El profesor dirá: "uno, dos, rojo" (o cualquier otro color). En este momento todos elevan el paracaídas y aquellos cuyo color coincide con el nombrado cambian de sitio por debajo del paracaídas y antes de que éste se desinfle. Para ello deben pisar sólo dentro de los aros. Si alguno pisa fuera o si el paracaídas, al desinflarse, toca alguna parte de su cuerpo, se transforma en estatua de sal y, por lo tanto, no puede moverse. Se puede salvar a las estatuas de sal dándole un beso cuando que se realizan los cruces.

\section{A manera de conclusión}

Los seres humanos fuimos creados para vivir en comunidad, esto implica todos los momentos de nuestra vida. Al nacer, ir al preescolar, a la escuela, al colegio, como padres, etc. somos parte de un mundo inmerso en un proceso de transformación constante. Todo esto no sería posible sin la cooperación, elemento que implica apoyar, motivar, ayudar, ser solidario y comprensivo.

Mediante la práctica de actividades lúdicas el valor de la cooperación se ve favorecido en las interrelaciones, donde ponemos en práctica como maestros reconocer el papel que cada persona tiene, sus actitudes positivas y negativas; así como se crean ambientes de confianza y compañerismo.

Es a través de nuestra experiencia en la implementación de actividades de este tipo y con materiales como los paracaídas, que proponemos una estrategia pedagógica simple que involucra directamente a los niños y niñas en el tema de los valores y del respeto de unos y otros.

\section{Referencias bibliográficas}

Bolaños, R. Taller de Paracaídas. Escuela Educación Física y Deportes. Universidad de Cosa Rica. San José. 2000.

"Cooperación". Obtenido el 8 de julio del 2004, de http:/www.livingalues.net/ español/valores.htm

Díaz, A. et al. Desarrollo Curricular para la Formación de Maestros Especialistas en Educación Física. España: Editorial Gymnos. 1993.

Flinchun, B. "Early Childhood Movement Programs. Preparing Teachers for Tomorrow". Journal Physical Education, Recreation and Dance. 59(7): 62-67. 1988.

Hetzer, H. El juego y los Juguetes. Argentina: Editorial Kapeluz. 1992.

Krauss, R. Recreation and Leisure in Modern Society. HarperCollins Publishers ( $4^{a}$ edición). 1990. 
Lacayo, M. y Coello, L. Educación Física, Deporte y Recreación al Alcance de Todos. Honduras: Talleres de NICOP. 1992.

Lázaro, A. "Radiografía del Juego en el Marco Escolar". En: Psicomotricidad. Revista de Estudios y Experiencias. $\mathrm{N}^{\mathrm{o}}$ 51, vol.3. pp.7-22. 1995.

"Los Valores" Obtenido el 17 de diciembre del 2004, de http:/www.ecojoven.com/ uno/05/valores2.html

Ministerio de Educación Pública. Programa de Estudios: Ciclo de Transición de la Educación Preescolar. Costa Rica: Ministerio de Educación Pública. 1996.

Orlick, T. Libres para Cooperar. Libres para Crear. Barcelona: Paidó Tribo. 1990.

Orlick, T. Juegos y Deportes Cooperativos. España: Popular. 1986.

Osorio, M. "El Juego". Obtenido el 10 de julio del 2004, de http://galeon.com/ educacion_fisica/investigaciones. html 2000.

Pérez, E. "Juegos Cooperativos: Juegos para el Encuentro". En: Lecturas de Educación Física y Deportes. Año 3, $\mathrm{N}^{\circ} 9$. Buenos Aires. Obtenido el 26 de junio del 2004, de http://www.efdeportes.com/efd9/jue.htm 1998.

Ríos, M. "Los Juegos Sensibilizadores: Una Herramienta de Integración Social". En: Apuntes. Revista de Educación Física y Deportes. (38), 93-98. 1994.

"Valores" Obtenido el 17 de diciembre del 2004, de http:/www.grupas.org/valores.htm

Vygotski, S. El Desarrollo de los Procesos Psicológicos Superiores. Barcelona: Grijalbo. 1979.

Zapata, O. El Aprendizaje por el Juego en la Etapa Maternal y Pre-Escolar. México: Editorial Pax. 1990. 
